\title{
Evacuateurs de Turkwel et de Katse
}

\author{
Turwel and Katse spillways
}

\author{
par P. Jehanno et C. Odeyer \\ Ingénieurs hydrauliciens \\ SOGREAH
}

This article presents the particular design features of the surface spillways and bottom outlets of Katse and Turkwel, two arch dams in Africa. For the bottom outlets, the design involves throttling the water stream at the outlet by converging side walls, allowing the impact zone to be spread out over a wider area and further from the dam, thereby improving energy dissipation. For the Katse surface spillway $(170 \mathrm{~m}$ head), the design includes a very long chute ( $30 \mathrm{~m}$ head), accelerating the water to very high speeds to spread the stream flow and move the jet impact further away from the base of the dam. Improved aeration combined with flow dividing blocks increase water stream break-up and jet atomisation. The combination of these specially designed systems enhances energy dissipation and minimises scour effects downstream of the dams.

\section{O OUVRAGES ÉVACUATEURS DU BAR- RAGE DE TURKWEL (KENYA)}

\subsection{Introduction}

Le barrage de Turkwel, mis en service en 1991, est situé sur la rivière Turkwel, à $180 \mathrm{~km}$ en amont de son embouchure dans le lac Turkana et à $400 \mathrm{~km}$ au N/N-O de Nairobi, au Kenya.

Les buts de cet aménagement sont la production d'énergie et l'irrigation.

Les chiffres clés de cet aménagement sont les suivants :

- type de barrage

: voûte à double courbure,

- hauteur maximale

: $155 \mathrm{~m}$,

- longueur en crête

: $170 \mathrm{~m}$

— volume de béton du barrage

: $160000 \mathrm{~m}^{3}$

- volume de la retenue

: 1,65 milliards de $\mathrm{m}^{3}$,

- centrale souterraine équipée de : 2 groupes Francis de $53 \mathrm{MW}$ chacun,

- débit de l'évacuateur de surface : $1470 \mathrm{~m}^{3} / \mathrm{s}$,

- débit du pertuis de fond : $515 \mathrm{~m}^{3} / \mathrm{s}$.

La particularité du site est l'étroitesse de la gorge où est implanté le barrage : au niveau de la rivière, la largeur de la gorge est d'environ $50 \mathrm{~m}$. Sur chaque rive, la rivière est bor- dée par des falaises quasi verticales. Les ouvrages d'évacuation devaient être conçus pour éviter d'injecter des pressions dans les fissures existant sur chaque rive, principalement en rive droite. L'impact des jets provenant des ouvrages d'évacuation devait donc éviter les falaises en aval du barrage.

L'évacuateur de surface et le pertuis de fond ont été mis au point sur un modèle réduit à fond affouillable, à l'échelle du $1 / 70$.

\subsection{Evacuateur de surface}

L'évacuateur de surface est de type à surface libre, non vanné. Il est constitué de 4 passes de $15 \mathrm{~m}$ de large. Son débit maximal est de $1470 \mathrm{~m}^{3} / \mathrm{s}$ (pour un niveau d'eau de $1154,8 \mathrm{~m})$.

Le seuil de chaque passe est arasé à la cote $1150 \mathrm{~m}$.

Chaque passe est constituée d'un seuil profilé de $5 \mathrm{~m}$ de haut (charge maximale en bas du coursier : $9,8 \mathrm{~m}$ ), avec des angles variables de sortie des jets $\left(+30^{\circ}, 0\right.$ et $-26^{\circ}$ par rapport à l'horizontale). L'objet de ces angles de sortie variables est double :

- éviter les impacts sur les falaises rive droite et rive gauche, ce qui oblige à concentrer en largeur les jets, la forme en voûte favorisant d'ailleurs cette concentration en largeur des écoulements, 
- étaler en longueur l'impact des jets pour limiter les profondeurs d'érosion en aval du barrage.

Pour éviter complètement des impacts de jets sur la falaise rive droite, un mur déflecteur a été installé sur la passe la plus proche de la rive droite.

\subsection{Evacuateur de fond}

Il est constitué d'un conduit de $28 \mathrm{~m}$ de long, équipé d'une vanne secteur de $4 \mathrm{~m}$ de large par 3,6 m de haut. La charge maximale sur la vanne est de $85 \mathrm{~m}$.

Afin d'éviter tout impact du jet sortant du pertuis de fond sur les falaises rive droite et rive gauche, l'extrémité du pertuis est équipé d'un pincement vertical installé à l'extrémité du pertuis, la largeur du pertuis passant de $4 \mathrm{~m}$ à $3,2 \mathrm{~m}$ sur une longueur de $2 \mathrm{~m}$.

Ce pincement entraîne un jet en forme de "lame de sabre ", qui étale la lame d'eau sur une grande longueur et permet ainsi un allongement de l'impact, ce qui a pour conséquence, d'une part d'éloigner l'impact du jet du barrage, d'autre part de permettre une dissipation sur une grande longueur, limitant ainsi les affouillements en aval du barrage.

Au débit maximal $\left(515 \mathrm{~m}^{3} / \mathrm{s}\right)$, le jet s'étale de $120 \mathrm{~m}$ à $190 \mathrm{~m}$ du barrage.

Les leçons tirées des essais du pertuis de fond de Turkwel ont été mises en application lors de la conception des pertuis de fond du barrage de Katse.

\section{II $\square$ OUVRAGES ÉVACUATEURS DU BAR- RAGE DE KATSE (LESOTHO)}

\subsection{Introduction}

Le barrage réservoir de Katse, mis en service en 1998, est l'une des composantes du vaste projet à buts multiples du " LESOTHO HIGHLANDS", au Lesotho, pays de 30355 $\mathrm{km}^{2}$, enclavé au sud-est de l'Afrique du Sud.

Le barrage est situé sur la rivière Malibamatso, à 100 km environ à l'est de Maseru, capitale du Lesotho.

Le but principal du barrage de Katse est d'assurer le transfert d'eau vers le bassin de la rivière Vaal, en République d'Afrique du Sud.

Le projet du " LESOTHO HIGHLANDS" est un projet destiné à développer par phases successives les ressources en eaux des montagnes du Lesotho, à l'aide d'une série de barrages, tunnels, stations de pompage et aménagements hydroélectriques.

Ses objectifs principaux sont les suivants :

- détourner les eaux s'écoulant naturellement dans une direction sud-ouest du Lesotho vers le nord, en direction de centres habités en République d'Afrique du Sud,

- produire de l'énergie électrique au Lesotho, en utilisant la chute disponible des eaux dérivées,

- permettre l'alimentation en eau, l'irrigation et le développement régional au Lesotho.

Le projet est prévu d'être construit en quatre phases principales et devrait s'achever vers 2020.

La première partie de la phase $\mathrm{n}^{\circ} 1$ est actuellement achevée ; elle est principalement constituée des travaux ou structures suivants :

- les routes d'accès et les travaux d'infrastructure,
- le barrage de Katse, qui constitue le réservoir principal de l'ensemble du projet,

- la prise d'eau de transfert (hauteur : $80 \mathrm{~m}$, diamètre : $10,5 \mathrm{~m}$ ), située à $20 \mathrm{~km}$ en amont du barrage de Katse,

- le premier tunnel de transfert (longueur : $45 \mathrm{~km}$, diamètre : $4,35 \mathrm{~m}$ ), du barrage de Katse à l'usine hydroélectrique de Muela,

- la première centrale hydroélectrique de Muela et le barrage associé (hauteur : $60 \mathrm{~m}$ ),

- le premier tunnel d'alimentation (longueur : $35 \mathrm{~km}$, diamètre : $4,3 \mathrm{~m}$ ).

\subsection{Généralités sur le barrage de Katse et les modèles réduits}

Les caractéristiques principales du barrage de Katse et des ouvrages annexes sont les suivantes :
- type de barrage
: voûte à double courbure,
- hauteur maximale
: $185 \mathrm{~m}$
- longueur en crête
: $710 \mathrm{~m}$,
— volume de béton
: $2320000 \mathrm{~m}^{3}$,
- évacuateur de surface
: 10 passes de $16 \mathrm{~m}$ de large (arasées à la cote $2053 \mathrm{~m}$ ), débit maximal : $6080 \mathrm{~m}^{3} / \mathrm{s}$ (niveau de retenue : $2060 \mathrm{~m}$ ),

- 2 pertuis de fond de débit unitaire $415 \mathrm{~m}^{3} / \mathrm{s}$ (sous la cote maximale de retenue hors crue : $2053 \mathrm{~m}$ ),

— volume de la retenue : 1,95 milliard de $\mathrm{m}^{3}$,

— contre barrage aval : situé à $250 \mathrm{~m}$ du barrage de Katse, longueur en crête : $232 \mathrm{~m}$,

- longueur du seuil $\quad: 70 \mathrm{~m}$, hauteur : $37,5 \mathrm{~m}$, cote du seuil : $1908,2 \mathrm{~m}$

Le barrage de Katse constitue un record en Afrique en termes de hauteur et de volume pour un barrage en béton.

La conception des ouvrages évacuateurs et la hauteur du contre barrage aval ont été en partie dictés par la qualité du rocher situé au pied de la voûte, à savoir :

- entre la cote $1893 \mathrm{~m}$, qui est le niveau du lit de la rivière naturelle, et $1888 \mathrm{~m}$, le rocher est sain, mais légèrement fissuré, les micro-fissures produisant des blocs d'environ $0,3 \mathrm{~m}$ de dimension,

- entre $1888 \mathrm{~m}$ et $1885 \mathrm{~m}$, le rocher est massif et très peu fissuré,

- entre $1885 \mathrm{~m}$ et $1881 \mathrm{~m}$, le rocher est constitué d'une couche de brèches de faible qualité,

- sous la cote $1881 \mathrm{~m}$, le rocher est à nouveau massif.

Afin d'éviter des problèmes de glissement dû à la couche de brèches, il fut décidé que la couche de rocher massif situé entre les cotes $1888 \mathrm{~m}$ et $1885 \mathrm{~m}$ située sur la couche de brèches ne devait pas être touchée par les effets de la dissipation d'énergie des ouvrages évacuateurs.

Pour l'évacuateur à surface libre, 3 solutions ont été proposées (voir fig. $\mathrm{n}^{\circ} 1$ ) :

- La solution $n^{\circ} 1$ dite " courte" : cette solution est constituée d'un seuil déversant réduit au minimum et implique une fosse de dissipation revêtue, 


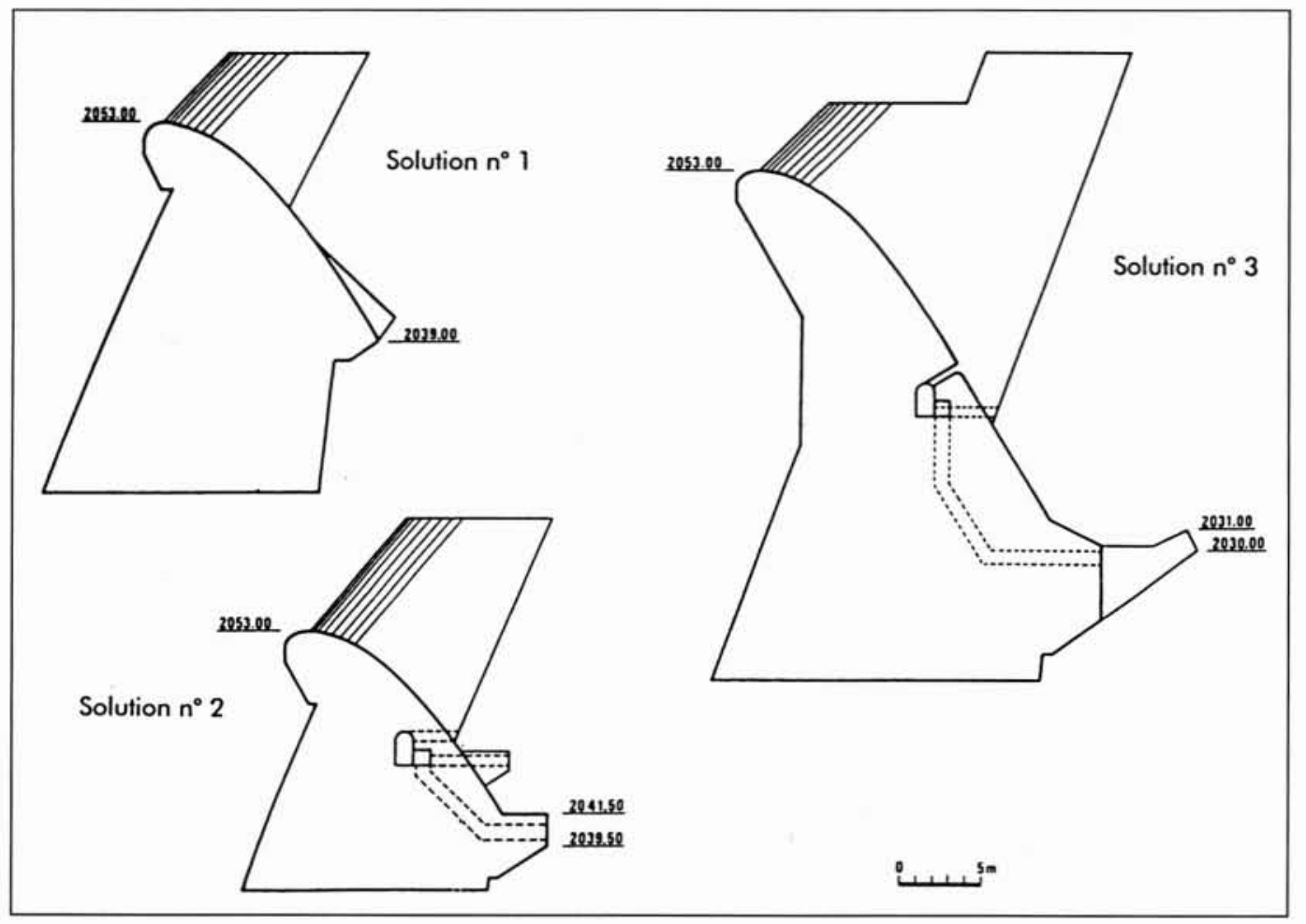

1. Barrage de

Katse : formes

d'évacuateur envisagées.

- La solution $\mathrm{n}^{\circ} 2$ dite " intermédiaire" : cette solution est de type " Colonel Robert", utilisée sur plusieurs barrages en Afrique du Sud, ainsi qu'au barrage Victoria, au Sri Lanka. La chute pour ces différents barrages est de l'ordre de $80 \mathrm{~m}$, donc nettement moindre que celle du barrage de Katse (170 m environ).

Cette solution est basée sur le fractionnement de l'écoulement à l'aide de blocs spéciaux ("Splitter blocks "). Ces blocs, placés sur la face aval du seuil, casse le jet et crée un effet de rotation au moment de la séparation des lames d'eau. Quand l'écoulement atteint le déflecteur horizontal placé quelques mètres plus bas, l'effet de rotation permet d'améliorer l'étalement et le fractionnement des lames d'eau.

- La solution $n^{\circ} 3$ dite " longue " : pour cette solution, l'idée principale est de prendre le maximum de vitesse afin de pouvoir étaler en longueur les lames d'eau à l'aide de déflecteurs. Elle implique un coursier assez long pour prendre suffisamment de vitesse et de n'avoir sur les déflecteurs que de faibles lames d'eau faciles à diriger.

Seules les solutions $n^{\circ} 2$ et 3 ont été retenues et ont fait l'objet d'études sur modèle réduit.

La comparaison des solutions $\mathrm{n}^{\circ} 2$ et 3 , ainsi que l'étude d'un des 2 pertuis de fond ont été effectuées à l'aide d'un modèle à grande échelle (1/30), pour prendre en compte les phénomènes d'entraînement d'air et d'atomisation des jets.
Pour des raisons de coût et de délais, il n'était pas envisageable de représenter la totalité de l'évacuateur de surface sur le modèle au 1/30.

Seules ont été représentées sur le modèle au 1/30 trois passes sur dix (en fait 2 passes complètes et deux demipasses).

Une fois mis au point les évacuateurs de surface et de fond sur le modèle au 1/30, l'ensemble des autres tests (comme par exemple les phases de travaux et le contre barrage aval) fut effectué sur un modèle général à l'échelle du 1/70.

\subsection{Mise au point de l'évacuateur de surface}

L'objet de cette étude a consisté à comparer les 2 solutions d'évacuateur envisagées, en améliorant chaque solution d'évacuateur pour limiter les érosions en aval du barrage.

Les principaux débits sont décrits par le tableau $n^{\circ} 1$.

Pour chaque solution, 2 séries de tests ont été menées :

— une première série (voir para. 2.3.1 et 2.3.2), en considérant un contre barrage aval constitué d'un seuil de $150 \mathrm{~m}$ de large arasé à la cote $1910 \mathrm{~m}$. A l'aval de la voûte était disposé du matériau affouillable arasé à la cote $1890 \mathrm{~m}$. Cette série a permis de mettre au point pour chaque solution les formes d'évacuateur permettant de limiter au maximum les affouillements en aval du barrage principal,

\begin{tabular}{|c|c|}
\hline Débit $\left(\mathrm{m}^{3} / \mathrm{s}\right)$ & Caractéristiques \\
\hline 6080 & PMF sans évacuateur latéral ${ }^{(*)}$ \\
\hline 4929 & PMF avec évacuateur latéral de $250 \mathrm{~m}$ \\
\hline 4010 & crue de 1000 ans \\
\hline 3320 & crue de 500 ans \\
\hline
\end{tabular}

${ }^{(*)}$ : évacuateur en col déversant dans une vallée latérale.
Tableau $n^{\circ} 1$ : Principaux débits testés 
— une seconde série (voir para. 2.3.3), destinée à déterminer le niveau minimal aval nécessaire pour éviter les érosions de la couche de rocher située entre les cotes 1885 et 1888 , ceci afin de définir les caractéristiques du contre barrage aval, permettant de former un matelas d'eau amortisseur.

\subsubsection{Mise au point de la solution $n^{\circ} 2$}

La disposition initiale de l'évacuateur comportait un coursier équipé de blocs "splitter" et terminé par une plateforme horizontale située $18,5 \mathrm{~m}$ sous le niveau maximal de retenue.

Pour le débit maximal $\left(6080 \mathrm{~m}^{3} / \mathrm{s}\right)$ et la solution initiale, la profondeur d'érosion maximale était de $15 \mathrm{~m}$ sous une cote initiale de $1890 \mathrm{~m}$ (soit une nappe d'eau de $37,5 \mathrm{~m}$ ), le point d'érosion maximale étant situé à $80 \mathrm{~m}$ du barrage.

Pour $4929 \mathrm{~m}^{3} / \mathrm{s}$, la nappe d'eau au point d'érosion maximale était également de 37,5 m (profondeur de l'érosion : $16 \mathrm{~m}$ ).

L'observation des écoulements sur l'évacuateur ont amené à améliorer la solution en modifiant certains dispositifs :

- en utilisant des “ splitters " plus étroits et inclinés vers l'aval, afin d'obtenir une distribution des débits plus uniforme,

- en plaçant la plate-forme aval plus bas, afin d'augmenter la vitesse d'éjection de l'écoulement et d'éloigner ainsi le point d'impact des jets dans la fosse aval,

- en prolongeant la plate-forme horizontale de $2 \mathrm{~m}$ au droit de l'espace situé entre les " splitters ", afin d'augmenter l'étalement et le fractionnement de l'écoulement.

Avec la solution améliorée, la profondeur d'érosion maximale dans la fosse aval a pu être limitée par rapport à la solution initiale : $10 \mathrm{~m}$ de moins pour $4900 \mathrm{~m}^{3} / \mathrm{s}$ et $5 \mathrm{~m}$ de moins pour le débit maximal $\left(6080 \mathrm{~m}^{3} / \mathrm{s}\right)$. L'érosion maximale se situe à environ $80 \mathrm{~m}$ du barrage, et ce pour des débits de $3320 \mathrm{~m}^{3} / \mathrm{s}$ à $6080 \mathrm{~m}^{3} / \mathrm{s}$.

\subsubsection{Mise au point de la solution $n^{\circ} 3$}

Cette solution se composait initialement d'un coursier long, équipé :

- d'une rainure d'aération, nécessaire compte tenu de la charge maximale du coursier, et permettant la dispersion de l'écoulement par atomisation des lames d'eau,

- de dents situées à l'extrémité du coursier, à raison de 2 dents par pertuis. L'angle de sortie de l'écoulement à l'extrémité des dents est de $+25^{\circ}$ par rapport à l'horizontale. Dans l'espace entre les dents, il est de $-30^{\circ}$.

L'objet de ces 2 angles de sortie différents est d'étaler en longueur l'écoulement. De plus les dents permettent un écoulement en nappe qui favorise la dispersion.

La dispersion des jets n'est possible que si les vitesses sont grandes et l'épaisseur des lames d'eau faible (la chute sur les déflecteurs ou cuillers doit être en général supérieure à 4 fois la charge critique sur le seuil).
C'est pourquoi une chute importante $(30 \mathrm{~m}$ au maximum sur la partie horizontale des dents) avait été adoptée pour cette solution.

Pour le débit maximal $\left(6080 \mathrm{~m}^{3} / \mathrm{s}\right)$ et la solution initiale, l'érosion maximale était de $11 \mathrm{~m}$ (soit une nappe d'eau de $33 \mathrm{~m}$ ), le point d'érosion maximale étant situé à $140 \mathrm{~m}$ du barrage.

Pour $4000 \mathrm{~m}^{3} / \mathrm{s}$ (crue de 1000 ans), l'érosion maximale était de $5 \mathrm{~m}$, soit une nappe d'eau de $26 \mathrm{~m}$, avec un point d'érosion maximale situé à $120 \mathrm{~m}$ du barrage.

L'amélioration de cette solution d'évacuateur fut menée en suivant 2 voies :

- l'amélioration de l'entraînement d'air, afin d'augmenter l'atomisation des jets,

- l'installation de blocs en forme de coin, placés entre la rainure d'aération et l'extrémité aval du coursier, afin de favoriser la dispersion des jets.

Ces blocs constituent en fait une adaptation des “ splitters ” à un évacuateur à longue portée assurée par une chute importante.

L'amélioration de l'entraînement d'air a été assurée par une légère rampe située en amont de la rainure d'aération. Des blocs de dimensions modestes ont été rajoutés (largeur : $0,8 \mathrm{~m}$, longueur : $0,9 \mathrm{~m}$, hauteur : $0,4 \mathrm{~m}$ ), afin d'augmenter la surface d'échange. L'effet de ce dispositif est important pour les plus grands débits : $6 \mathrm{~m}$ de moins d'érosion pour $6080 \mathrm{~m}^{3} / \mathrm{s}$. Pour $3320 \mathrm{~m}^{3} / \mathrm{s}$, la différence d'érosion par rapport à la solution initiale reste modeste $(1 \mathrm{~m})$.

L'installation de blocs de fractionnement en aval permet d'améliorer encore les performances de l'évacuateur : à $3320 \mathrm{~m}^{3} / \mathrm{s}$, l'érosion est nulle. A 4000 et $5000 \mathrm{~m}^{3} / \mathrm{s}$, elle est d'environ $1 \mathrm{~m}$. A $6080 \mathrm{~m}^{3} / \mathrm{s}$, elle est de $2 \mathrm{~m}$ au maximum, à $140 \mathrm{~m}$ du barrage, avec une première érosion de $1 \mathrm{~m}$ située à $80 \mathrm{~m}$ du barrage.

On doit noter que la hauteur des blocs de dispersion est faible en comparaison des blocs initiaux de la solution $n^{\circ} 2$, dite "Colonel Robert " (dimension d'un bloc "disperseur" de la solution $\mathrm{n}^{\circ} 3$ après mise au point: hauteur : $0,8 \mathrm{~m}$, longueur : $2,2 \mathrm{~m}$, largeur $3 \mathrm{~m}$ ).

\subsubsection{Conclusions sur le choix de la solution d'évacuateur}

La seconde série de tests a été effectuée pour les 2 solutions d'évacuateur (après leur mise au point), afin de déterminer la profondeur minimale d'eau évitant toute érosion en aval. Les principaux résultats de ces tests sont résumés par le tableau 2.

On constate que la solution $\mathrm{n}^{\circ} 3$ permet de limiter de $7 \mathrm{~m}$ la hauteur du contre barrage aval. De plus, l'impact des jets où l'énergie est maximale, est situé bien plus en aval du barrage pour cette solution : $140 \mathrm{~m}$ pour l'impact maximal au lieu de $80 \mathrm{~m}$ pour la solution $\mathrm{n}^{\circ} 2$. Enfin, l'étalement des jets (mesuré à la cote $1912 \mathrm{~m}$ ) est de 60 à $80 \mathrm{~m}$ pour la solu-

Tableau $n^{\circ} 2$ : Profondeur d'eau minimale évitant toute érosion.

\begin{tabular}{|c|c|c|}
\hline Débit $\left(\mathbf{m}^{\mathbf{3}} / \mathbf{s}\right)$ & Solution $\mathbf{n}^{\circ} \mathbf{3}$ & Solution $\mathbf{n}^{\circ} \mathbf{2}$ \\
\hline 3300 & 18,5 & 20 \\
\hline 4900 & 25 & 28,7 \\
\hline 6080 & 27 & 34 \\
\hline
\end{tabular}


2. Evacuateur de surface de Katse (solution retenue). Détails.
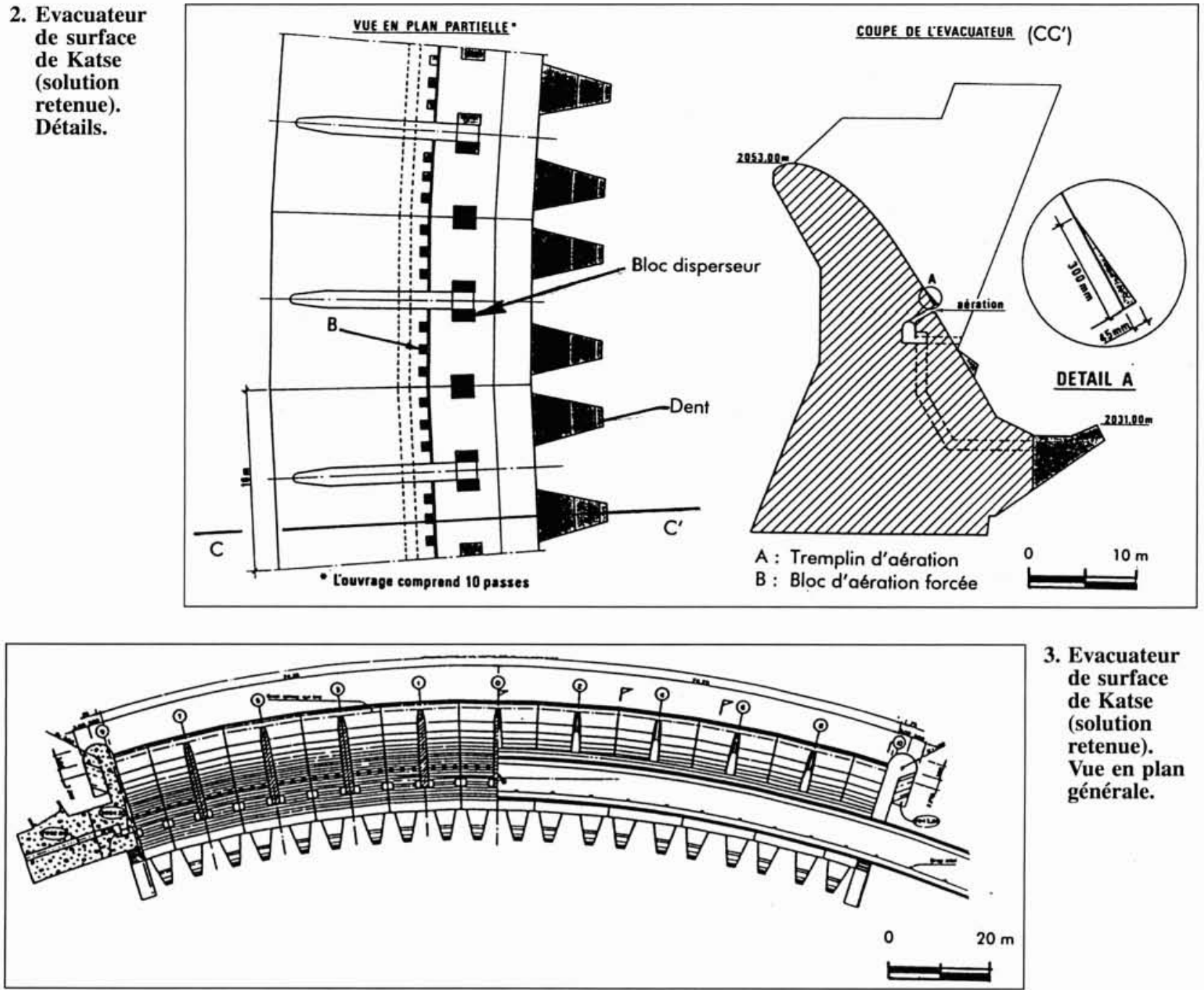

3. Evacuateur de surface de Katse (solution retenue). Vue en plan générale.

tion $\mathrm{n}^{\circ} 2$ et de 80 à $130 \mathrm{~m}$ pour la solution $\mathrm{n}^{\circ} 3$. Compte tenu de ces différents éléments, la solution $n^{\circ} 3$ fut finalement retenue pour le projet.

La solution d'évacuateur retenue est définie par les figures $\mathrm{n}^{\circ} 2$ et $\mathrm{n}^{\circ} 3$.

La coupe générale montrant le contre barrage et la fosse de dissipation est illustrée par la figure $\mathrm{n}^{\circ} 4$.

L'application de la formule de Veronese, en tenant compte de la concentration du jet due à l'effet de la voûte amène, pour $6080 \mathrm{~m}^{3} / \mathrm{s}$, à une profondeur variant de $56 \mathrm{~m}$ à $65 \mathrm{~m}$ suivant les hypothèses. La comparaison avec les $27 \mathrm{~m}$ obtenus avec la solution $\mathrm{n}^{\circ} 3$ permet d'apprécier l'efficacité de la solution retenue.

\subsection{Etude du pertuis de fond}

A Katse, chaque pertuis est d'une longueur totale de $50 \mathrm{~m}$ et est équipé d'une vanne secteur de $2,9 \mathrm{~m}$ de large par $3,4 \mathrm{~m}$ de haut (débit maximal : $415 \mathrm{~m}^{3} / \mathrm{s}$ ).

Compte tenu de la charge disponible $(117 \mathrm{~m})$, une solution proche de celle retenue pour le pertuis de fond de Turkwel a été adoptée : le jet est pincé dans un plan vertical, par un angle de $7^{\circ}$, sur $4 \mathrm{~m}$ de long. Le pincement permet d'allonger d'une manière significative la zone d'impact, permettant ainsi de limiter les effets de la dissipation d'énergie, c'est-àdire les affouillements et les courants de retour. De plus, la forme en pincement améliore les conditions propres à éviter la cavitation.

Tableau $n^{\circ} 3$ : Essais du pertuis de fond - Principaux résultats.

\begin{tabular}{|c|c|c|c|c|}
\hline Débit(m $\left.\mathrm{m}^{3} / \mathrm{s}\right)$ & $\begin{array}{c}\text { Distanced'impact } \\
(\mathrm{m})^{(*)}\end{array}$ & $\begin{array}{c}\text { Profondeur d'eau } \\
(\mathrm{m})^{(*)}\end{array}$ & $\begin{array}{c}\text { Profondeur d'érosion } \\
(\mathrm{m})\end{array}$ & $\begin{array}{c}\text { Impact des lames d'eau } \\
(\mathrm{m})\end{array}$ \\
\hline 400 & - & 15 & 0 & 70 à 200 \\
\hline 100 & 60 & 18,6 & $4,5^{(* *)}$ & 35 à 60 \\
\hline $50^{(* * *)}$ & 68 & 16,3 & 2,5 & - \\
\hline
\end{tabular}

(*) au point d'érosion maximale

$\left({ }^{* *}\right)$ sans prolongement du pincement et sans béquet, l'érosion était de $9 \mathrm{~m}$.

(***) ouverture partielle sous un niveau amont de $1947 \mathrm{~m}$. 


\section{Applications industrielles de la mécanique des fluides}

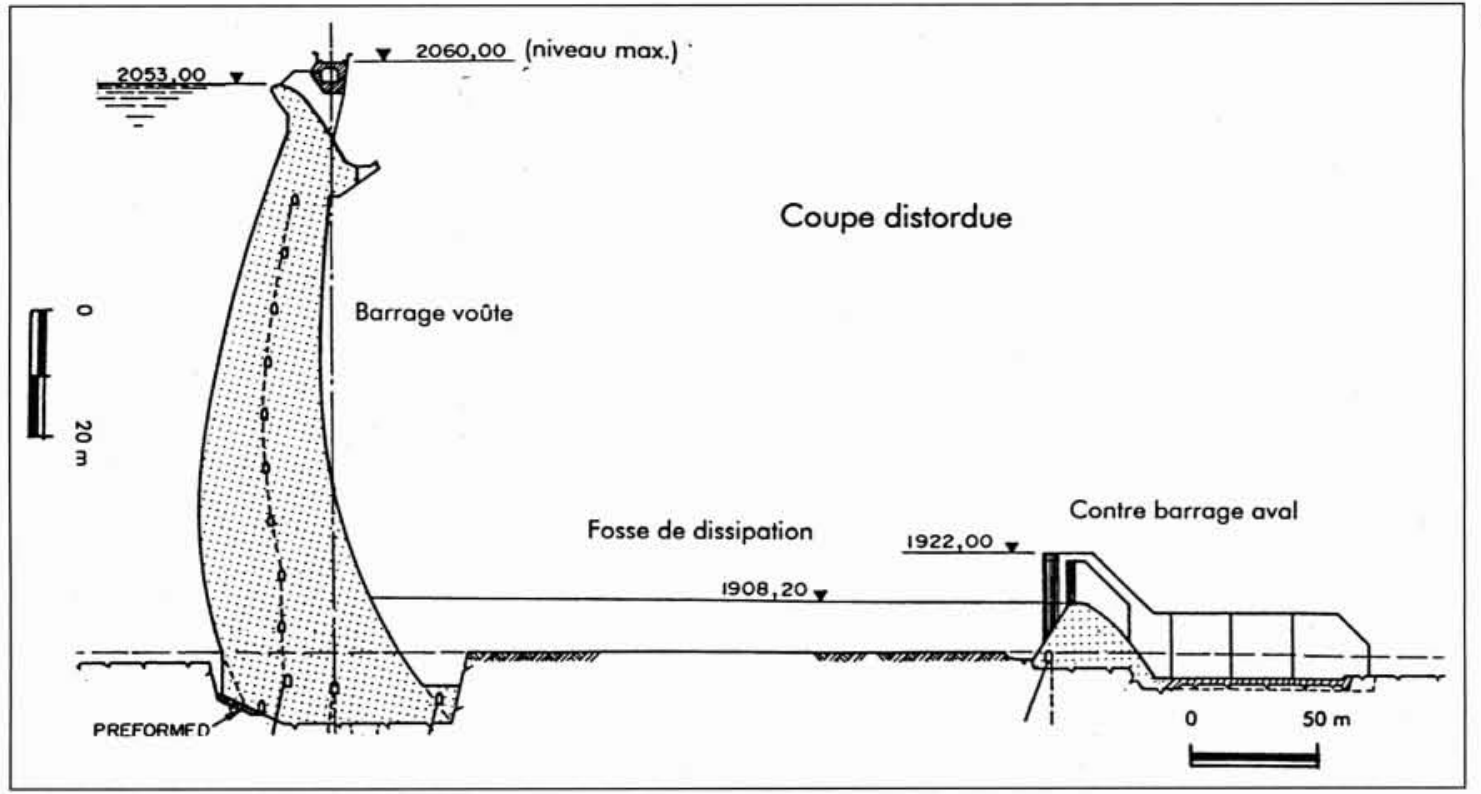

4. Barrage de Katse : coupe dans l'axe de la rivière.

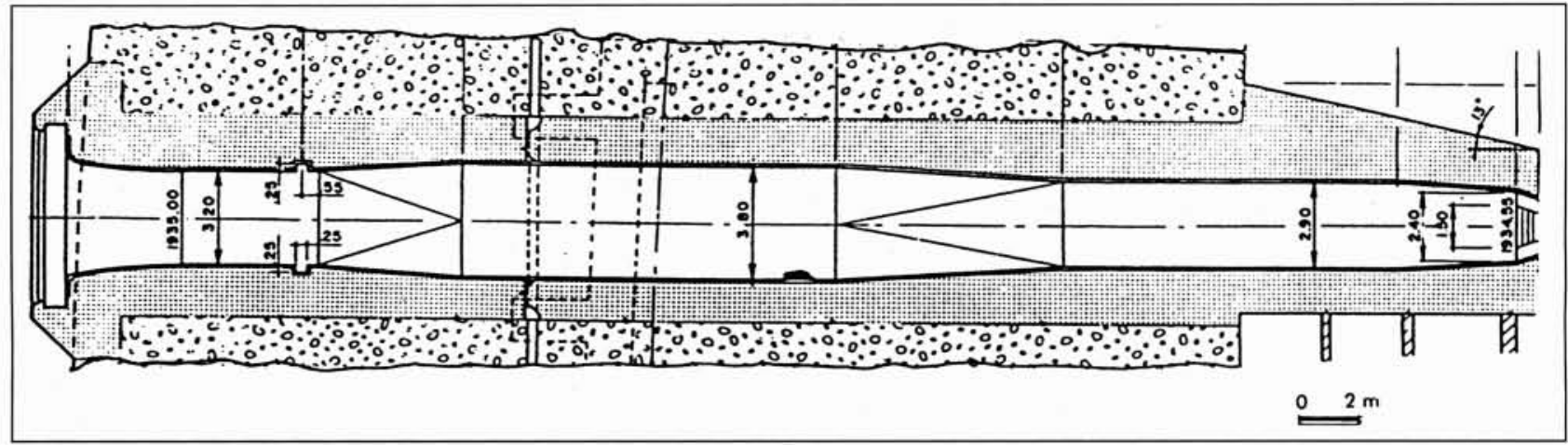

5. Pertuis de fond de Katse : vue en plan (coupe).

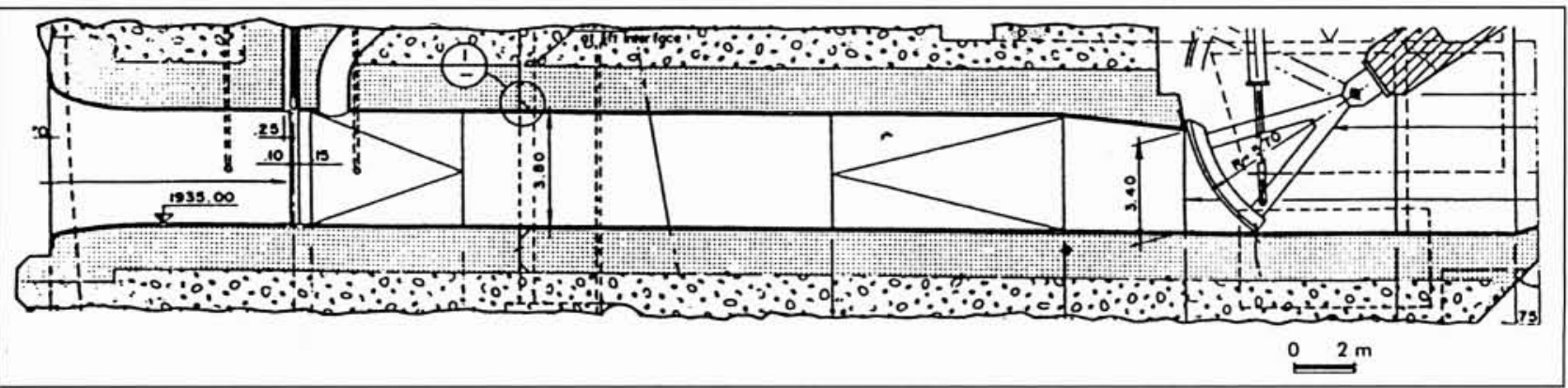

6. Pertuis de fond de Katse : coupe verticale (dans l'axe).

Sur la solution définitive, 2 dispositifs ont été ajoutés (voir fig. $\mathrm{n}^{\circ} 5$ et $\mathrm{n}^{\circ} 6$ ) :

- un pincement court $(0,75 \mathrm{~m})$ et plus aigu $\left(20^{\circ}\right)$, en prolongement du premier pincement,

- un " béquet" ou tremplin prolongeant la partie horizontale du pertuis, placé en surplomb, et de largeur égale à la moitié de celle du pertuis à son extrémité.

Le béquet permet d'étaler le jet aux débits intermédiaires et d'éviter les inconvénients d'une solution de type " cuiller" (ressaut, gel...).

Pour les essais du pertuis de fond, le niveau d'eau aval était maintenu en supposant un contre barrage aval de $80 \mathrm{~m}$ de large, avec une cote de seuil de 1903,4 m.

Les essais ont été effectués en général à pleine ouverture de vanne (voir résultats tableau $\mathrm{n}^{\circ} 3$ ).

Comme on peut le constater, l'étalement de la lame d'eau est important ( $70 \mathrm{~m}$ à $200 \mathrm{~m}$ au débit maximal), ce qui permet une bonne dispersion de l'énergie et donc de limiter les érosions.

Un des pertuis a fonctionné en 1997 à pleine ouverture et sous un niveau proche du niveau maximal $(2045 \mathrm{~m}$ pour un maximum de $2053 \mathrm{~m}$ ) pendant plusieurs jours sans problème. 